\title{
Study and Performance in a MIMO System to Receiving
}

\author{
Bouamama Réda Sadouki \\ Telecom laboratory \\ streetcaptain abdelhadi APC de Mérine \\ /sba/algéria
}

\begin{abstract}
Modern systems of Wireless Communications have limited capacity and processing techniques are required more sophisticated in order to improve system performance. One of the techniques in vogue is the application of MIMO (Multiple Input Multiple Output), known to improve the channel capacity and or the probability of bit error. The purpose of this paper is the comparison of two techniques for MIMO systems. The first technique using a series of antennas on the side of the terminal (and therefore much RF chain) is known to improve system performance and that there is a feedback loop containing the state of the channel or not. However, the use of these antennas on the terminal requires a lot of power because the remains of additional RF chains. The second one is for the terminal to choose the best antenna corresponding to the channel conditions. For the uplink, this requires a return to the channel state radio base station to mobile. The performance will always be suboptimal compared to using multiple channels in reception. But the terminal would need to maintain single RF chains which potentially save a lot of power.
\end{abstract}

\section{Keywords}

Minimum mean-squared error (MMSE), Maximum likelihood (ML),Bit error rate (BER), Successive interference cancellation (SIC), zero Forcing (ZF),Multiple-Input Multiple-Output ( MIMO).

\section{INTRODUCTION}

Digital technology completes the range of applications offered by the analog transmissions. Simpler and more robust than analog media, media digital communication already allow many services one of the main reasons for this delay is the willingness of manufacturers to integrate the latest technologies to increase substantially the spectral efficiency of mobile systems. One of them, combining multiple antennas for transmission and reception, is the subject of this thesis. Such multiple antenna systems, MIMO systems or (for Multi -Input MultiOutput), have the potential to significantly increase the capacity of wireless transmissionand, if they exist for more than a decade, experienced a marked revival. Several architecturesas have been developed in parallel, from the spatial multiplexing space-time codes, and all offering incredible performance improvement of transmission systems. However, this multi-antenna technology does not work in all environments, and must comply with many constraints. It is increasingly growing and affluent innovations. This work therefore aims firstly to raise some specific spatiotemporal systems such restrictions, and secondly to optimize their performance.

It also gives the main characteristics and limitations of multiantenna systems, before proposing the intended applications. Then we give the various diversity techniques that can combat fading, followed by a realistic modeling of the MIMO channel to become familiar with the spatial aspect brings an increase in the number of antennas, the capacity is one of the parameters most important, we present, therefore, the

\author{
Hichem CHAKER \\ Telecom laboratory \\ BP 285, 13400 GhazaouetTlemcen ALGERIA
}

capacity of SISO and MISO systems MIMO. And proposes to involve the efferent equalizer types used for antenna selection at receiver and the most performance and we propose a simulation of multi- antenna systems which allows both the calculated capacity of MIMO systems and evaluated the antenna bit error rate according to the number ( TEB / Ne) with equalization .

\section{MATHEMATICAL MODEL AND SIMULATION}

\subsection{Introduction}

Thesimulatedtransmission systemcorrespondsto the systemnumber ofstructuresforthereceiver $(2 \times 2)$ of the MIMO channel.

- WithSISOequalizer(ZF, MMSE, ML).

- $\quad$ 1-MIMO Equalizerzeroforcing(ZF).

- 2-MIMO EqualizerMinimum MeanSquareError (MMSE).

- 3-MIMO equalizerwith maximumlikelihood (ML), Maximum Likelihood(ML).

- 4-MIMO

withzeroforcingequalizerthesuccessiveInterferenceCan cellationZF-SIC withoptimal control.

- The5-MIMO withMMSE equalizerwithSICandoptimal control.

\subsection{Sisowith equalizer(ZF, MMSE, ML)}

Now we considerthe casewherethere is an "input signaloutputsignal") SISOsystem.Forthis reason,we will try tomakethe "RBA" biterror rateunderthe application ofequalization(ZF, ML, MMSE) at the receiver. We assume that thechannelisRayleighandwe usetheBPSKchannel.

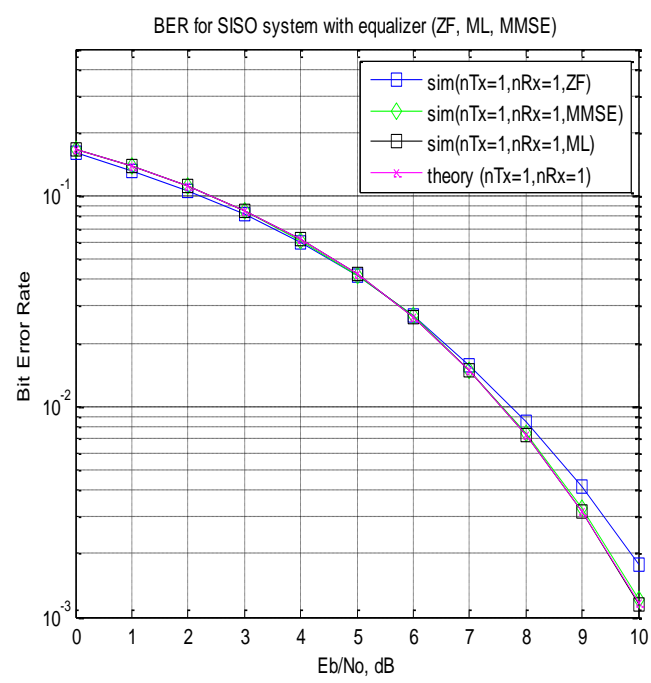

Fig 1: BERforSISOsystemwith equalizer(ZF, ML, MMSE).

Observations 
1/As desired, the simulation results show good agreement with theory.

2/simulation results show that $\mathrm{ZF}$ and MMSE equalizer and ML gives the same ( bit error rate ) "BER ". Finally, I 'm not sure whether we can call it as the MMSE equalization «ZF, ML» as there is no interference terms. Weconclude thatthe application ofequalization( $Z F, M M S E, \quad M L)$ in a system"SISO" gives the same "BER". sincethey are thesamenErr, The problem isthe increase in noiseforbadsubchannels. Thediversity orderis, however,MRMT 1(which impliesthat there isno diversityifMR =MT)

\subsection{MIMOwithZero Forcingequalizer(ZF)}

We now considerthe casewhere there is aformation of aMultiple

InputMultiple Output(MIMO)antennamultisystem.Forthis reason,we will limitour discussionto2transmitand 2 receiveantennas(whichhas $2 \times 2$ MIMOchannel). We assume that thechannelisRayleightragicandmulti-chain we useBPSKmodulation.

ForaMIMOsystem(2, 2), theprobableuseof a device fortwotransmission antennasisas follows:

Consider that we havea sequence oftransmissionexe $\left\{x_{1}, x_{2} x_{3}, \ldots, x_{n}\right\}$.

- Under normaltransmissionAvonsendus $x_{1}$ firsttime interval, $\boldsymbol{x}_{2}$ the2ndtime interval...etc.

- However,aswe now have twotransmit antennas,we can groupthe symbols ina set of twoin the firsttime intervalwe send $x_{1}$ and $x_{2}$ andhavethrough thetwoantennas $1, \quad 2, \quad$ respectively, in the 2 ndinterval,send $x_{3}$ and $x_{4}$ crossesDeusantennas 1,2 respectively, and so follows.

- Note that since we are grouping two symbols and send in a time interval, we need $\frac{n}{2}$ time interval to complete the transmission - data rate is doubled!

- This is the simple explanation of a probable MIMO transmission with two transmit antennas and two receiving antennas.

\subsubsection{The application of $(Z F)$ for $M I M O(2,2)$}

We will now tryto understandmathematicallyhowto extract thetwo symbolsthat interferedwith other[5]. Thesignalsreceived atthe firstreceiving antennaandthefollowingform:

$$
\begin{aligned}
& y_{1}=h_{1,1} x_{1}+h_{1,2} x_{2}+n_{1}=\left[h_{1,1} h_{1,2}\right]\left[\begin{array}{l}
x_{1} \\
x_{2}
\end{array}\right]+ \\
& n_{1} .(1)
\end{aligned}
$$

Thesignalsreceived on thesecondreceiving antennaandthefollowingform:

$$
y_{2}=h_{2,1} x_{1}+h_{2,2} x_{2}+n_{2}=\left[h_{2,1} h_{2,2}\right]\left[\begin{array}{l}
x_{1} \\
x_{2}
\end{array}\right]+
$$$$
\boldsymbol{n}_{2} \text {. (2) }
$$

Or: $\boldsymbol{y}_{1}, \boldsymbol{y}_{\mathbf{2}}$ the Symbolsreceived at the firstandthesecondreceiving antennarespectively.

$h_{1,1}$ is the channel for the first antenna transmitting the worm first receiving antenna.

$h_{1,2}$ is the channel for antenna 2 nd worm issue the first receiving antenna.

$h_{2,1}$ is the channel for the first transmit antenna worm 2 nd the receiving antenna.

$h_{2,2}$ is the channel for transmit antenna 2 nd worm 2 nd the receiving antenna.

$x_{1}, x_{2}$ are two symbols transmitted.

$n_{1}, n_{2}$ Is the Pruitt on the two receiving antennas?

We assume that $h_{1,1}, h_{1,2}, h_{2,1}, h_{2,2}$ known and the receiver also knows $\boldsymbol{y}_{1}$ and $\boldsymbol{y}_{2}$ then the unknown is $x_{1}$ and $x_{2}$ two equations and two unknowns may have solved? Yes.

For further understanding, the above equation can be represented in matrix notation as follows:

\subsubsection{The equivalent form:}

$\boldsymbol{y}=\boldsymbol{H x}+\boldsymbol{n}$.

To resolve, we know that we need to find a matrix $W$ that $\operatorname{check} \boldsymbol{W} \boldsymbol{H}=\boldsymbol{I}$. To meet this constraint the linear detector Zero forcing $(\mathrm{ZF})$ is given by:

$W=\left(H^{H} H\right)^{-1} H^{H}$.

This matrix is also known as the pseudo inverse in general.(Calculated as the inverse matrix $H^{H} H^{-1}$ ).

With use of the zero forcing equalizer (ZF), the receiver can obtain an estimate of these two symbols transmitted $x_{1}, x_{2}$ is to say:

$\left[\begin{array}{l}\widehat{x}_{1} \\ \widehat{x}_{2}\end{array}\right]=\left(H^{H} H\right)^{-1} H^{H}\left[\begin{array}{l}y_{1} \\ y_{2}\end{array}\right]$

BER for BPSK modulation with 2x2 MIMO and ZF equalizer (Rayleigh channel)

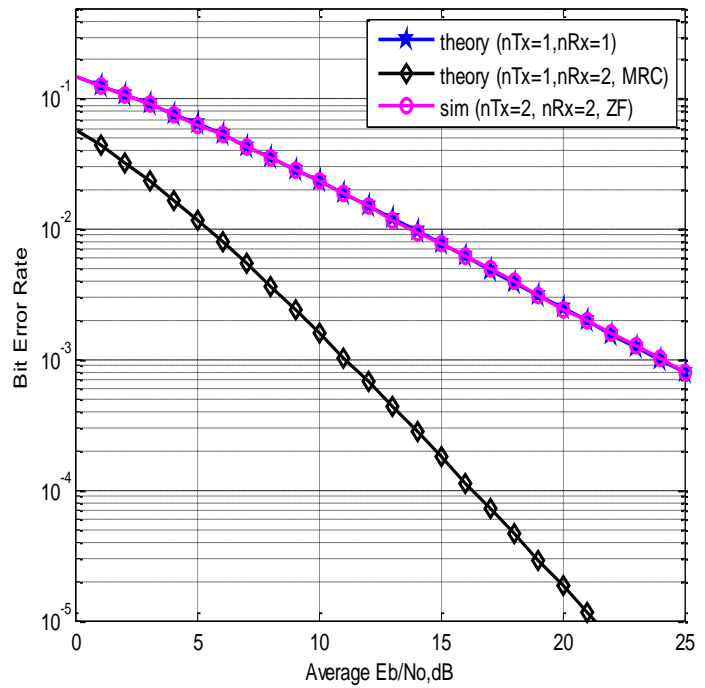

Fig 2: BERperformancefor $2 \times$ 2MIMOchannelwithZFequalizer.

As expected, the simulated results with the MIMO system (2 $\times 2)$ using the BPSK modulation in a Rayleigh channel shows that the results obtained in the matching as a system $(1 \times 1)$ to the BPSK channel el Rayleigh.

\subsection{MIMO with MMSE equalizer}

2.4.1 The application of (MMSE) for the MIMO system (2.2) 
We will now try to understand mathematically how to extract the two symbols that interfered with others. The signals received at the first receiving antenna and the following form:

$$
\begin{array}{ll}
-y_{1}=h_{1,1} x_{1}+h_{1,2} x_{2}+n_{1}=\left[h_{1,1} h_{1,2}\right]\left[\begin{array}{l}
x_{1} \\
x_{2}
\end{array}\right]+ \\
n_{1} .
\end{array}
$$

The signals received on the second receiving antenna and the following form:

$$
\begin{array}{ll}
y_{2}=h_{2,1} x_{1}+h_{2,2} x_{2}+n_{2}=\left[h_{2,1} h_{2,2}\right]\left[\begin{array}{l}
x_{1} \\
x_{2}
\end{array}\right]+ \\
n_{2} .
\end{array}
$$

Or: $y_{1}, y_{2}$ are

thesymbolsreceivedonthefirstandthesecondreceiving antennarespectively.

- $\boldsymbol{h}_{1,1}$ thechannel for thefirstantennatransmittingthewormfirstreceiving antenna.

- $h_{1,2}$ is the channel forantenna $2{ }^{\text {nd }}$ wormissuethefirstreceiving antenna.

- $h_{2,1}$ is the channel for thefirstantennatransmittingtheworm $2^{\text {nd }}$ receiving antenna

- $h_{2,2}$ is the channel forantenna $2^{\text {nd }}$ worm transmission $2^{\text {nd }}$ thereceiving antenna

- $\quad x_{1}, x_{2}$ twosymbolstransmitted

- $\quad n_{1}, n_{2}$ IsthePruitton the tworeceiving antennas?

We assume that $h_{1,1}, h_{1,2}, h_{2,1}, h_{2,2}$ are known andthe receiveralso knows $y_{1}$ and $y_{2}$ thenthe unknown is $x_{1}$ and $x_{2}$, twoequations andtwo unknownsmayhavesolved?yes. For convenience, theabove equationcan be representedinmatrix notationas follows:

\subsubsection{The matrix Equivalent}

$\boldsymbol{y}=\boldsymbol{H} \boldsymbol{x}+\boldsymbol{n}$.

The Minimum Mean Square Error approach (MMSE) sought to find a coefficient $W$ that minimizes the following criterion:

$E\left\{[W y-X][W y-x]^{H}\right\}$

$W=\left[H^{H} H+N_{0} I\right]^{-1} H^{H}$.

When comparing the equation Zero Forcing equalizer, apart from the expression $N_{0} I \quad$ I both equations are comparable. Indeed, when the noise term is zero, the MMSE equalizer reduced to zero forcing equalizer.

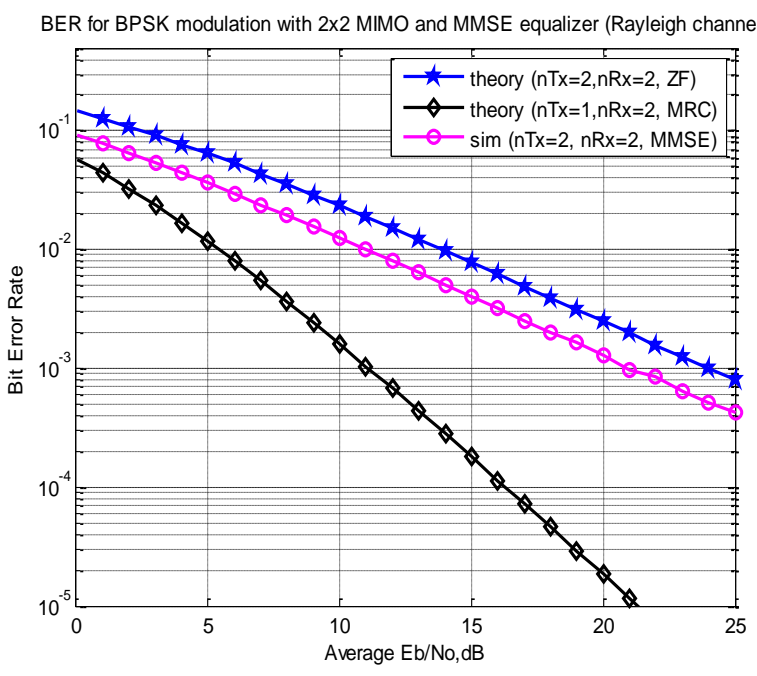

Fig 3:BERperformanceforMIMO $(2 \times 2)$ withMMSE equalizer.

TheMMSEachievesa compromise betweennoise reductionand interference. Thediversity orderis identical to theZF. Compared to theZero Forcingequalizer(ZF) at $10^{-3} \mathrm{BER}$, we can see thattheminimummean square error(MMSE)equalizerresults innearly 3dBimprovement.

\subsection{MIMOequalizerML(maximum likelihood)}

We will now tryto understandmathematicallyhowto extract thetwo symbolsthat interferedwith others.

Thesignalsreceived atthe firstreceiving antennaandthefollowingform:

$y_{1}=h_{1,1} x_{1}+h_{1,2} x_{2}+n_{1}=\left[h_{1,1} h_{1,2}\right]\left[\begin{array}{l}x_{1} \\ x_{2}\end{array}\right]+n_{1} \cdot(10)$

The signals received on the second receiving antenna and the following form:

$$
y_{2}=h_{2,1} x_{1}+h_{2,2} x_{2}+n_{2}=\left[h_{2,1} h_{2,2}\right]\left[\begin{array}{l}
x_{1} \\
x_{2}
\end{array}\right]+n_{2}
$$

Or: $y_{1}, y_{2}$ sont les Symboles reçu sur la première et la deuxième antenne de réception respectûverinent.

- $h_{1,2}$ is the channel for thefirstantenna of thewormissuethefirstreceiving antenna.

- $h_{2,1}$ is the channel for thefirstantennatransmittingtheworm $2^{\text {nd }} \quad$ receiving antenna

- $h_{2,2}$ is the channel forantenna $2^{\text {nd }}$ wormtransmission $2^{\text {nd }}$ thereceiving antenna

- $x_{1}, x_{2}$ aretwosymbolstransmitted.

- $\quad n_{1}, n_{2}$ IsthePruitton the tworeceiving antennas?

We assume that $h_{1,1}, h_{1,2}, h_{2,1}, h_{2,2}$ known andthe receiveralso knows $y_{1}$ and $y_{2}$ thenthe unknown is $x_{1}$ and $x_{2}$, deux twoequations andtwo unknownsmayhavesolved?Yes.

For convenience, theabove equationcan be representedinmatrix notationas follow:

$y=H x+n$. 


\subsubsection{Other assumptions ML (maximum likelihood)} The channel is flat fading - In simple terms, this means that the multi- channel has a single tap. Thus the convolution operation reduces to a simple multiplication. For a more thorough discussion on the Flat fading and frequency selectivity, it was admitted that: - Channel use each transmit antenna is independent of the chain by other antennas sent.

- For $\boldsymbol{i}^{\text {th }}$ transmitting antenna $j^{\text {th }}$ worm receiveantenna, each symbol transmitted is multiplied by a random variable complex $h_{j, i}$. As the channel under consideration is a Rayleigh channel, the real and imaginary parts $h_{j, i}$ are Gaussian distributed

An average $\quad \mu_{h_{j, i}}=0$ AndVariance $\quad \sigma_{h_{j, i}}^{2}=\frac{1}{2}$.

- examine the chain between each transmit antenna and reception (issued and received) is an independent random time varying.

- On the receiving antenna, the noise $\mathrm{n}$ characterize the Gaussian probability density function $p(n)$ with: $p(n)=\frac{1}{\sqrt{2 \pi \sigma^{2}}} e^{\frac{-(n-\mu)^{2}}{2 \sigma^{2}}}(12)$

with

$\mu=0$ and $\sigma^{2}=\frac{N_{0}}{2}$.

- The channel $h_{j, i}$ is known at the receiver.

\subsubsection{Receiver maximum likelihood (ML):}

The maximum likelihood receiver tries to find $\widehat{\boldsymbol{x}}$ which minimizes

$J=|\mathcal{Y}-H \hat{x}|^{2}(4.13)$

Since theBPSKmodulation, the possible valuesare+1 or $1 x_{1}$ sameas $x_{2}$ prendrevalues+1or- 1 , And for the solutionof maximum likelihood, we need to find the minimumof allfourcombinations $\boldsymbol{x}_{\mathbf{1}}$ and $\boldsymbol{x}_{2}$.

The estimation oftransmittedsymbols ischosen according tothe minimum valueof these four valuesisto say:

- Iflistheminimumone,

$\mathcal{J}+1,+1 \Rightarrow[11]$ (4.10)

Iftheminimumis $\mathcal{J}+1,-1 \Rightarrow\left[\begin{array}{ll}1 & 0\end{array}\right]$. (4.11)

- Ifthe minimum is $\mathcal{J}-1,+1 \Rightarrow[0$ 1]. (4.12)

- Ifthe minimum is $\mathcal{J}-1,-1 \Rightarrow[00] .(4.13)$

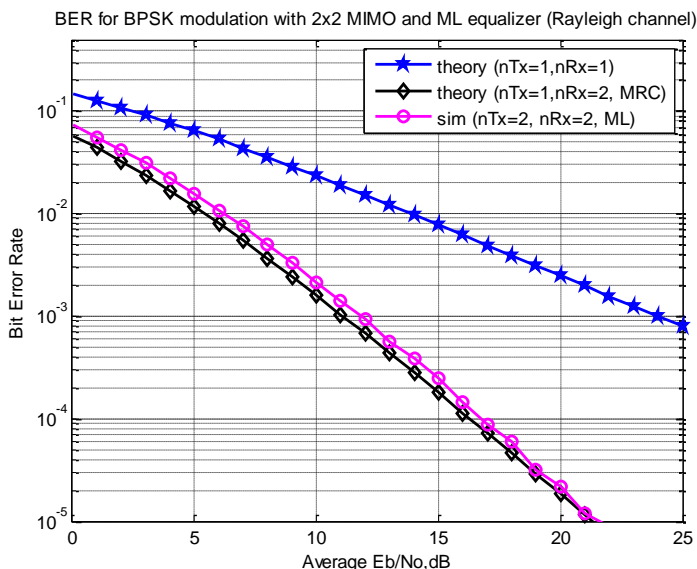

Fig 4: BER performance for $2 \times 2$ MIMO equalizer with Maximum Likelihood (ML).
The results of $(2 \times 2)$ MIMO equalizer with Maximum Likelihood (ML) helped to achieve a performance close to the corresponding system (1.2), one transmit antenna and two receive antennas in the case (MRC).

If we use a higher order such as 64QAM constellation, then the calculation of the maximum likelihood equalizer could become too complex. 64QAM with 2 spatial streams and we need to find the minimum of $64^{2}=4096$ combinations

\subsection{MIMO zero forcing equalizer with sic and optimal control (ZF - SIC)}

We will explore a variant of ZF -SIC called zero forcing successive interference cancellation with optimal control. We assume that the channel Rayleigh multipath and flat-fading channel and BPSK modulation.

BER for BPSK modulation with 2x2 MIMO and ZF-SIC equalizer (Rayleigh channel)

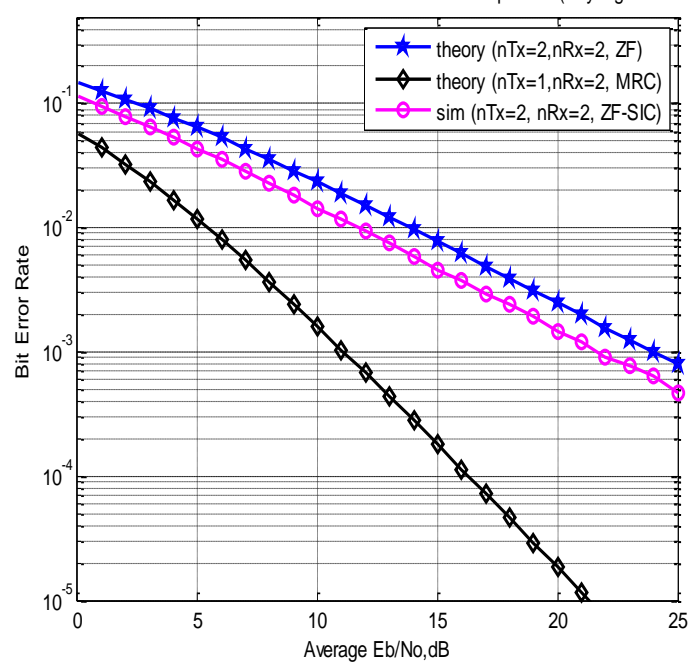

Fig 5: BER performance for $2 \times 2$ MIMO channel equalized (ZF-SIC).

To finish the graph if we compare the two equalization criteria: zero forcing (ZF) and (ZF-SIC), the data adds the result of improvements near 4.0dB SNR optimal control corresponding to the point $\mathrm{BER}=10^{-3}$.

\subsection{MIMO with MMSE-SIC and optimalcontrol}

We extend the notion of (MMSE-SIC) called Minimum Mean Square Error successive interference cancellation with optimal control and simulate their performance. We assume that the channel Rayleigh multipath and flat-fading channel and BPSK modulation. 


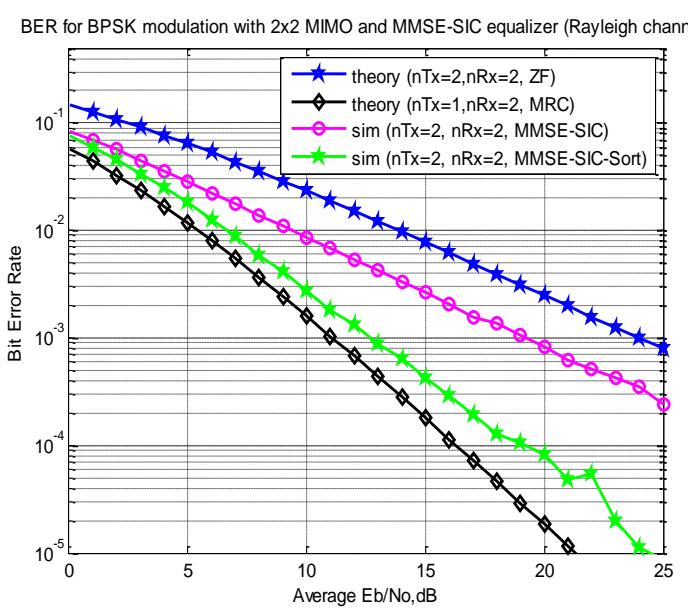

Fig6: BER for $2 \times 2$ MIMO channel with the equalizer (MMSE -SIC ) with and without optimal ordering ).

To finish the graph if we compare the two criteria equalization, Minimum Mean Square Error (MMSE) and (MMSE -SIC) successive interference cancellation easy, adds the given optimal control results near $5.0 \mathrm{~dB}$ improvements SNR corresponding to BER $=1 \mathrm{O}^{-3}$.

Performance is now closely match the curve of the case (MRC) (1.2) with 1 transmit antenna and 2 receive antennas. The curve with the BER for the ZF equalizer MIMO channel $(2 \times 2)$ is identical to the curve of BER for a system $(1,1)$ to receive one transmit antenna 1 ( SISO ).

The MMSE equalization successive interference cancellation SIC with optimal batch performance that slightly poor rather than ML command.

\subsection{The probability of error as a function of SNR (BER / SNR)}

The bit error rate for BPSK with AWGN is calculated the following way.

$$
p_{b} \frac{1}{2} \operatorname{erfc}\left(\sqrt{\frac{2 E_{b}}{N_{0}}}\right) .
$$

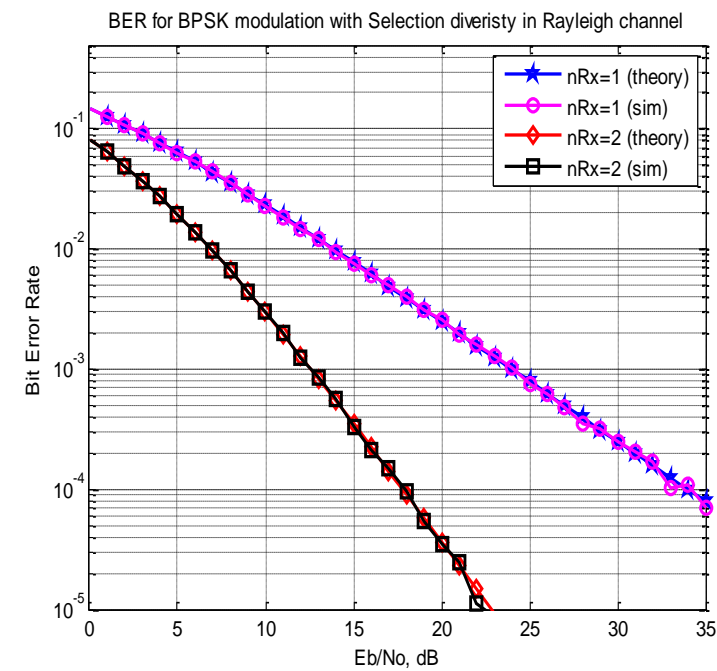

Fig 7: BERperformance for Rayleigh canalwithreceiving antenna (diversity).

As desired, the simulation results show good agreement with theory.Primer graph with two (2) a receiving antenna selection diversity.It was a round $16 \mathrm{~dB}$ improvement in point $\mathrm{BER}=$
$10^{-4}$.

\subsection{Evaluation of BER in an AWGN channel with $\mathrm{Nr}$ receive antennas}

2.9.1 Case of a single antenna transmission Let us begin the discussion with an antenna (1) transmission, sending signals with energy $\mathrm{v}$ and a receiving antenna.Since we are considering only the BPSK modulation, the signals that are sent is $+\sqrt{E_{b}}$ and. $-\sqrt{E_{b}}$ When there to be a single receiving antenna with a thermal noise (AWGN) with average $\mu=0$ and variance $\sigma^{2}=\frac{N_{0}}{2}$. La probability density function of the noise is: $p(n)=\frac{1}{\sqrt{2 \pi \sigma^{2}}} e^{\frac{-(n-\mu)^{2}}{2 \sigma^{2}}}$.

The received signal is of the form: $y=x+n$ Where:

- $\quad y_{\text {is }}$ the received symbol.

- $\quad x$ is the transmitted symbol (taking values $+\sqrt{E_{b}}$ and $-\sqrt{E_{b}}$ ).

- $\quad n$ The additive white Gaussian noise $(A W G N)$.

Our discussion for BPSK in AWGN, we know that the probability of bit error is : $p_{b}=\frac{1}{2} \operatorname{erfc}\left(\sqrt{\frac{E_{b}}{N_{0}}}\right)$.

\subsubsection{Case with two (02) of transmit antennas}

Now, consider the case where we have two receiving antennas each having noise ( AWGN ) with average $\mu=0$ and variance $\sigma^{2}=\frac{N_{0}}{2}$. As the noise on each antenna is independent of each other, in the signal processing, we can say that the noise on each antenna are That is to say, independent and identically distributed. The transmitter is still sending power $\operatorname{symbols} E_{b}$

The received signal is of the form $\left[\begin{array}{l}\boldsymbol{y}_{1} \\ \boldsymbol{y}_{2}\end{array}\right]=x+\left[\begin{array}{l}\boldsymbol{n}_{1} \\ \boldsymbol{n}_{2}\end{array}\right]$

with :

- $y_{1}, y_{2}$ are symbols of receive antenna received 1,2 , respectively.

- $y_{1}, y_{2}$ are symbols of receive antenna received 1,2 , respectively.

- $\boldsymbol{x}$ Is the transmitted (taking is values $+\sqrt{E_{b}}$, est $-\sqrt{E_{b}}$, s) symbol

- $\quad \boldsymbol{n}_{1}, \boldsymbol{n}_{2}$ is the additive white Gaussian noise ( AWGN ) on the receiving antennas 1,2 respectively.

For simplicity, we assume that the signal $+\sqrt{E_{b}}$ was transmitted. At the receiver, we now have:

$y_{1}=\sqrt{E_{b}}+n_{1}$ and $y_{2}=\sqrt{E_{b}}+n_{2}$

To decode the simplistic (and better in this case) is to take the average of $\mathcal{Y}_{1}$ and $\mathcal{Y}_{2}$ and make the hard decision decoding, that is to say $y_{s}=\frac{y_{1}+y_{2}}{2}$ and if $y_{s} \geq 0$ implies that if the transmitted bit is $1 \quad$ and $\mathcal{Y}_{s} \leq 0$ Implies that the bit transmitted and equals 0 . Now, let us know if there is a gain by the reception diversity. Performing this average. $\mathcal{Y}_{s}$ Splitting in terms of signal and noise: 


$$
\begin{aligned}
Y_{s} & =\frac{\sqrt{E_{b}}+n_{1}+\sqrt{E_{b}}+n_{2}}{2} . \\
& =\sqrt{E_{b}}+\left(\frac{n_{1}+n_{2}}{2}\right) .
\end{aligned}
$$

(a)from the discussion on the sum of Gaussian random variables :

The first term of the above equation is the term that still transmits the signal symbols with energy $E_{b}$. Thesecond is the average of two. Terms of Gaussian noise.

If $X$ is a Gaussian random variable with mean $\mu_{1}$, and variance $\sigma_{1}{ }^{2}$ independent,$\quad Y$ is another Gaussian random variable with mean $\mu_{2}$, and variance $\sigma_{2}^{2}$, then $(X+Y)$ another random variable has a Gaussian average $\left(\mu_{1}+\right.$ $\left.\mu_{2}\right)$ and variance $\left(\sigma_{1}^{2}+\sigma_{2}^{2}\right)$

(b) from the discussion on the functions of Gaussian random variables

If $X$ is a Gaussian random variable with mean $\mu_{1}$, and variance $\sigma_{1}^{2},(a X+b)$ there is another Gaussian random variable with mean $\left(a \mu_{1}+b\right)$ Fromvarianceand $\left(a \sigma_{1}\right)^{2}$. Using the two equations above, the noise term $\frac{n_{1}+n_{2}}{2}$ is another Gaussian random variable with mean. $\mu=0$ and variance $\sigma^{2}=$ $\frac{N_{0}}{4}$.

In comparison with the same antenna, we can see that the variation of the duration of the noise is reduced by a factor of two 2. This implies that energy efficiency noise ratio of the two receiving antennas is two times the rate of energy to noise if a single antenna is

$\left[\frac{E_{b}}{N_{0}}\right]_{e f f, 2}=\frac{2 E_{b}}{N_{0}}(16)$

Thus, the probability of error for a case of two receive antennas is:

$p_{b}=\frac{1}{2} \operatorname{erfc}\left(\sqrt{\frac{2 E_{b}}{N_{0}}}\right)(17)$

The expression in decibels, with two receiving antennas, we only need to $10 \log _{10}(2)=3 d B$ the lowest energy $E_{b} /$ bit.

\subsubsection{Case with $N$ receiving antenna, the received} symbols are:

$\left[\begin{array}{c}y_{1} \\ y_{2} \\ \vdots \\ y_{N}\end{array}\right]=x+\left[\begin{array}{c}n_{1} \\ n_{2} \\ \vdots \\ n_{N}\end{array}\right](18)$

Or:

$\mathcal{y}_{1}, \mathcal{Y}_{2}, \ldots \ldots, \mathcal{Y}_{N}$ are the received symbols for the receiving antennas $\quad 1, \quad 2, \quad 3 \ldots \mathrm{N}, \quad$ respectively. $x$ is the transmitted symbol (taking ' is value $+\sqrt{E_{b}}$ and $\left.-\sqrt{E_{b}}\right)$ and $n_{1}, n_{2}, \ldots, n_{N}$ Is the additive white Gaussian noise ( AWGN ) on the receiving antennas $1.2,3, \ldots \ldots ., \mathrm{N}$, respectively, for demodulation, one calculates $y_{s}$ which is the average of all the $\mathrm{N}$ received symbols, is $\mathcal{Y}_{s} \geq 0$ if the transmitted bit is 1 , is so $\mathcal{Y}_{s} \leq 0$ implies that the bit 0 is transmitted

The variance of the expression $\frac{\left(n_{1}+n_{2}+\cdots+n_{N}\right)}{N}$ is the noise $: \frac{\sigma^{2}}{2 N}$.

The signal to noise ratio bit Effective $\mathrm{N}$ receiving antenna is $\mathrm{N}$ times the signal to noise ratio for a single antenna (single)

$\left[\frac{E_{b}}{N_{0}}\right]_{\text {eff }, N}=\frac{N E_{b}}{N_{0}}(19)$
Thus, the error probability of the $\mathrm{N}$ bits receiving antenna is : $p_{b}=\frac{1}{2} \operatorname{erfc}\left(\sqrt{\frac{N E_{b}}{N_{0}}}\right) \cdot(20)$

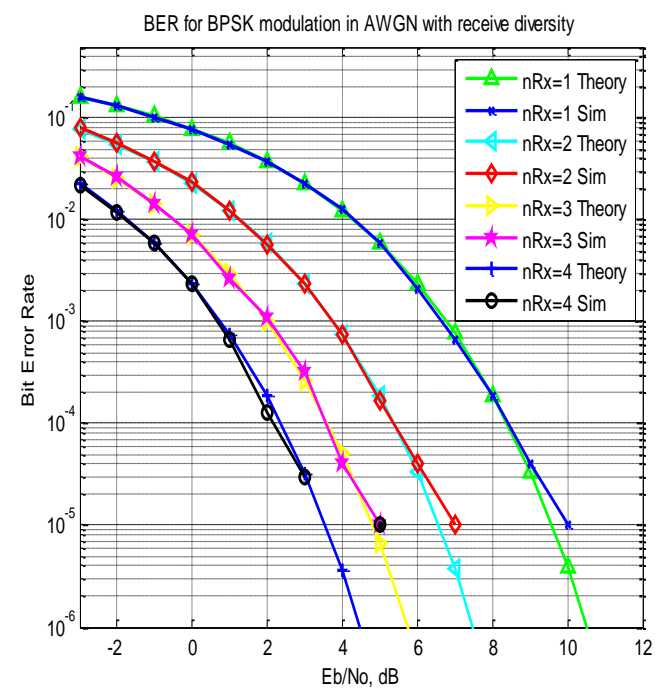

Fig8: Comparison of BER performance in an AWGN channel with diversity reception (number of receiving antennas $=1,2,3,4)$.

As desired, the simulation results show good agreement with theory.

With a system with $\mathrm{N}$ receive antenna gain relative to the case of a single receive antenna is about $10 \log _{10}(N)$. For example, in the figure above, for the bit error rate of $10^{-4}$.

With two receiving antennas lowest $\frac{E_{b}}{N_{0}}$ is $3 \mathrm{~dB}$.

with three receiving antennas lowest $\frac{E_{b}}{N_{0}}$ is $4.7 \mathrm{~dB}$

With four receiving antennas lowest $\frac{E_{b}}{N_{0}}$ is $6 \mathrm{~dB}$.

Hopefully this has helped you start with diversity reception.

\section{CONCLUSION}

The multi- antenna systems (MIMO) are booming thanks to their potential in terms of throughput and robustness to fading channel. The cost of production of complex systems is high. It is therefore interesting to predict, before completion, the important parameters to have the best performance. Throughout this paper, we studied the performance of MIMO systems equalized reception thanks to the knowledge of the channel (CSI). Precoding gives an extra freedom makes possible the optimization of transmission according to certain criteria (capacity, quality of service, etc). For these systems, we have established theoretically their bit error probability (PEB) for a Rayleigh channel, and bit error rate (BER) through simulations differ. We could then identify key parameters. We have focused on the selection of antenna reception (which is a technique provided by MIMO) and in particular the implementation of an important technique that offers the possibility of correcting the receivedsignals. This technique and call it EQ Based on the following, the transmission channel and rethinking impulse is why we gave them a large portion of this work. Then, as an application of equalization, we proposed a MIMO system with two transmit antennas and two receiving antennas, Finally, simulations 
have shown that the use of deferent types of equalization with MIMO technique helps to increase the bit rate and the correction of the channel effect .

\section{REFERENCES}

[1] G. J. Foschini. Layered space-time architecture for wireless communication a fading environment when using multi-element antennas. Bell Labs Technical Journal, pages 41-57, autumn 1996.

[2] N. Seshadri and C. E. W. Sundberg. Multi-level trellis coded modulations forthe Rayleigh fading channel. IEEE Transactions on Communications, 41(9):1300- 1310, September 1993.

[3] G. Ganesan and P. Stoica. Space-time diversity using orthogonal and amicable orthogonal designs. Wireless Personal Communications, 18(2):165-178, August 2001.

[4] V. Tarokh, H. Jafarkhani, and A. R. Calderbank. Spacetime block codes from orthogonal designs. IEEE Transactions on Information Theory, 45(5):1456-1467, July 1999.

[5] G. Ganesan and P. Stoica. Space-time diversity using orthogonal and amicable orthogonal designs. Wireless Personal Communications, 18(2):165-178, August 2001.

[6] C. Berrou, A. Glavieux, and P. Thitimajshima. Near Shannon limit error-correcting coding and decoding: Turbo-codes. In Proceedings of the IEEE International Conference on Communications (ICC), pages 10641070, Genève, Suisse, May 1993.
[7] A. S. Stefanov and T. M. Duman. Turbo coded modulations for systems with transmits and receive antenna diversity. In Proceedings of IEEE Global Telecommunications Conference (Globecom), pages 2336-2340, Rio De Janeiro, Brazil, 1999.

[8] K. Cavalec-Amis. Optimisation des Turbo Codes pour les systèmes à grande efficacité spectrale. $\mathrm{PhD}$ thesis, ENST Bretagne, 2001.

[9] A. Grant and C.Schlegel. Differential turbo space-time coding. In Proceedings of the IEEE Information Theory Workshop (ITW), pages 120-122, Cairns, Australia, September 2-7 2001

[10] C. Schlegel and A. Grant. Concatenated space-time coding. In Proceedings of the IEEE Symposium on Personal, Indoor and Mobile Radio Communications (PIMRC), pages 139-143, San Diego (CA), USA, September 30 - October 32001.

[11] B Hassibi and B. M. Hochwald. Cayley differential unitary space-time codes. IEEE Transactions on Information Theory, 48(6):1485-1503, June 2002.

[12] J.M. BROSS IER. Edition hermès, Signale et communication numérique Égalisation et synchronization.

[13] J. Labat, O. Macchi, Une structure universelle pour l'égalisation numérique autodidacte, GRET SI 97, Grenoble, 1997. 